African Crop Science Journal by African Crop Science Society is licensed under a Creative Commons Attribution 3.0 Uganda License. Based on a work at www.ajol.info/ and www.bioline.org.br/cs DOI: https://dx.doi.org/10.4314/acsj.v28i2.4

\title{
PRATIQUES CULTURALES ET DIVERSITE DES LIGNEUX COMPAGNES DANS LES EXPLOITATIONS CACAOYERES ET HEVEICOLES AL'EST DE LA CÔTE D'IVOIRE
}

\author{
M.D. KOUGBO ${ }^{1}$, D.F. MALAN ${ }^{1,2}$, M. DOGBA ${ }^{1}$ et A.S. KONAN ${ }^{1}$ \\ ${ }^{1}$ Université Nangui Abrogoua, Pôle de Recherche Environnement et Développement Durable / \\ Unité de Recherche Écologie et Biodiversité, 02 BP 801, Abidjan 02, Côte d'Ivoire \\ 2. Institut Botanique AKÉ ASSI d'Andokoi (IBAAN), 08 BP 172, Abidjan 08, Côte d'Ivoire
} Auteur correspondant : distelkougbo@gmail.com

(Received 10 January 2020; accepted 27 June 2020)

\section{RESUME}

En zone de forêt tropicale, les ligneux compagnes offrent de multiples services écosystémiques aux populations locales. Cependant, ils sont sur le point de disparaitre du fait que les agriculteurs ont désormais des objectifs tournés vers la production massive. Cette étude avait pour objectif d'analyser l'impact del'installation des exploitations cacaoyères et hévéicolessur la diversité de ces ligneux dansla région de l'Indénié-Djuablin en Côte d'Ivoire.Une enquête a été menée auprès de 108 agriculteurs (73 hommes et 35 femmes) de la région afin de connaître les processus et les activités relatifs à l'installation de leurs exploitations agricoles. Puis, la densité, l'aire basale et la diversité des ligneux compagnes ont étéévaluées à l'intérieur de 108 exploitations agricoles ( 54 cacaoyères et 54 hévéicoles) de différentes classes d'âge.Ceci a permis de comparerces parcelles agricoles à celles des forêts en reconstitution servant de témoins. Pour installer de nouvelles parcelles agricoles, ces agriculteurs réduisent la densité des arbresen fonction du type de culture et mettent le feu à la parcelle à exploiter. Ainsi lenombre moyen d'espèces de ligneux compagnesvarie de 4,92 à 6,17 espèces dans les exploitations à base de Theobroma cacao et de 2,08 et 2,50 espèces dans les exploitations à base de Hevea basiliensis. Toutefois, ces valeurs diffèrent de celle des forêts en reconstitution qui est en moyenne de 15,58 espèces.Contrairement aux exploitationsà base de Hevea brasiliensis, l'établissement des exploitations à base de Theobroma cacaoa eu comme conséquence une plus grande conservation de la diversité structurale et fonctionnelle des ligneux compagnes.

Mots Clés: Forêts, Hevea basiliensis, Theobroma cacao

\begin{abstract}
In tropical forests shade trees offer multiple ecosystem services to local populations. However, they are disappearing because farmers now have targets geared towards mass production. This study aimed at analysing the impact of cocoa and rubber farm establishments on the diversity of trees in the
\end{abstract}


Indénié-Djuablin region of Côte d'Ivoire. A survey was conducted among 108 farms in the region. Density, basal area and diversity of shade trees were evaluated in 108 farms (54 Theobroma cacao farms and 54 Hevea basiliensis farms). Farmers reduced the density of forest tree species depending on the type of crop established and residue management in the field. The average number of shade trees species left in Theobroma cacao farms ranged from 4,92 to 6,17 species and from 2,08 to 2,50 species for Hevea basiliensis farms. These values differed considerably from those of replanted forests, which averaged 15,58 species. The establishment of Theobroma cacao farms resulted in greater conservation of the structural and functional diversity of shade trees.

Key Words: Forests, Hevea basiliensis, Theobroma cacao

\section{INTRODUCTION}

Les ligneux compagnes sont des arbres, arbustes et arbrisseaux volontairement épargnés ou introduits par les agriculteurs dans leurs exploitations agricoles, et ce pour divers usages (Leakey, 1996). Cette pratique agricole qualifiée d'agroforesterie (Leakey, 1996) a été répanduedans le secteur agricole des pays tropicaux afin d'atténuer les effets néfastes découlant de la conversion des terres forestières en terres agricoles (Gockowski et Sonwa, 2010). Elle allie une production agricole durable à des mesures de conservation de l'environnement (Schroth et al., 2011). De ce fait, l'agroforesterie constitue un excellent compromis entre conservation de la biodiversité et lutte contre la pauvreté dans les régions tropicales (Deheuvels, 2011).

En effet, dans les paysages fortement anthropisés, les systèmes agroforestiers sont réputésabriter une diversité importante d'espèces de ligneuses compagnesparmi lesquelles figurent certaines considérées comme vulnérables ou menacées d'extinction (Asigbaaseet al., 2019; Temgoua et al., 2019). Dans le même temps, des recherches sur l'usage de ces ligneux ont montré que leur sélectionest fonction des besoins des agriculteurs. Ces derniers les maintiennent pour consommer ou vendre leurs fruits, soulager certaines pathologies, se bâtir un logement, pour en faire du combustible (Mangambu et al., 2012; Adou Yao et al., 2016) ou pour nourrir le bétail (Ngom et al., 2014). De plus, certains de ces ligneux sont épargnés ou introduits, car considérés comme bons indicateurs de changement de saison (Kouakou et al., 2018). Ils jouent également un rôle important dans la production agricolepar la fertilisation du sol et la création d'un ombrage suffisant pour la croissance des jeunes plants de cultures (Adou Yao et al., 2016). Au-delà de ces avantages,le maintien des ligneux compagnes contribue à atténuer les effets du réchauffement climatique en fixant le carbone atmosphérique dans leur biomasse (Folega $e t$ al., 2019; Giri et al., 2019).Ces arbres ont également un effet positif sur la dynamique de la régénération post-culturale, comme l'ont montré Carrière (2002), Sandor et Chazdon (2014) et Koffi et al. (2015).En dépit de la variété des services écosystémiques qu'offrent ces ligneux, leur survie demeure menacéedu fait essentiellement du changement de pratiques culturales (Siebert, 2002; Ruf, 2011). Tel est le cas dans les pays agricoles comme la Côte d'Ivoire, premier producteur mondialde fève de cacao et septième producteur mondial de caoutchouc.

En Côte d'Ivoire, les cacaoyers ont généralement été associés à d'autres ligneux dès l'installation de la plantation dans les premières grandes zones productrices de l'Est telles que la région de l'Indénié-Djuablin. Il a, d'ailleurs,été observé une reforestation d'environ 1000 hectares de forêt dans cette région (Koné et al., 2013). Toutefois, les exploitations cacaoyères y sont aujourd'hui vieillissantes, et, la forêt, antécédent cultural idéal, s'y faisant rare. Cette région a perdu 90 $\%$ du couvert forestier initial convertien 
exploitations cacaoyères et hévéicoles (Koné et al., 2014). Ainsi, pour s'adapter à la situation, certains agriculteurs remplacent les vieilles exploitations cacaoyères et les jachères par des exploitations hévéicoles (Ruf, 2012). D'autres par contre, procèdent à une régénération des vergers en utilisant des variétés de cacaoyers améliorés et à haut rendement qui ne tolèrent pas l'ombrage (Kpangui et al., 2015). Ce changement de pratiques culturales sous-entend un abattage quasi-total des ligneux compagnes dans les agrosystèmes. Pourtant, aucune étude à notre connaissance ne fait état de la survie de ces ligneux dans cette région de l'ancienne boucle du cacao.L'objectif de cette étude était d'analyser les pratiques culturales, la gestion et la diversité des ligneux compagnes dans les exploitations cacaoyères et hévéicoles de la région de l'Indénié-Djuablinà fin de gérer durablement ces ressources naturelles et leurs fonctions écologiques.

\section{METHODOLOGIE}

Zone d'étude. La région de l'Indénié-Djuablin est localisée dans la zone Est de la Côte d'Ivoire, entre les latitudes $5^{\circ} 53^{\prime}$ et $7^{\circ} 10^{\prime}$ Nord et les longitudes $3^{\circ} 10^{\prime}$ et $3^{\circ} 4^{\prime}$ ' Ouest (Fig. 1). Elle est soumise aux régimes climatiques de type subéquatorial (Koné et al., 2014) avec une précipitation moyenne annuelle de $118,50 \mathrm{~mm}$ etune température moyenne annuelle de $26,5^{\circ} \mathrm{C}$. La végétation originelle appartient au secteur mésophile du domaine Guinéen de Guillaumet et Adjanohoun (1971), précisément au type "forêt semi-décidue à Celtis spp. et Triplochiton scleroxylon K. Schum." (Koné, 2015). Toutefois, dans la pointe Sud de cette région, la végétation appartient au secteur ombrophile caractérisé par la présence de forêts denses humides sempervirentes à Eremospatha macrocarpa (Mann \& Wendl.) Wendl. et Diospyros mannii Hiern.

Collecte des données. La collecte des données a été effectuée dans les sous- préfectures de Yakassé-Fèyassé et de Zaranou, deux importantes localités de l'ancienne boucle du cacao, où des techniques de replantation cacaoyère sur de vieux vergers et l'adoption de l'hévéaculture ont été largement diffusées et pratiquées. Elle a d'abord commencé par l'inventaire des ligneux compagnes dans 108 exploitations agricoles dont 54 cacaoyères (Yakassé-Fèyassé $: \mathrm{n}=27$; Zaranou : $\mathrm{n}=27$ ) et 54 hévéicoles (Yakassé-Fèyassé : $\mathrm{n}=27$; Zaranou : $n=27)$. Pour ce faire, dans chaque localité et chaque type d'agrosystème, ces exploitations agricoles ont été réparties en raison de 9 exploitations suivant le stade de développement des plants de culture (moins de 5 ans ; 5-15 ans et plus de 15 ans). Une parcelle d'une superficie de $50 \mathrm{~m} \times 50 \mathrm{~m}$ a été ensuite disposée à l'intérieur de chacune des exploitations agricoles et tous les individus ligneux de plus de $2,5 \mathrm{~cm}$ de diamètre qui s'y trouvaient, à l'exception des plants de culture, ont été identifiés et comptés. Des forêts en reconstitution âgées de plus de 20 ans, servant de témoins ont également fait l'objet d'inventaire. Il s'agit de la forêt classée de Brassué à Yakassé-Fèyassé et quelque jachère à Zaranou. Dans ces formations végétales, 18 autres parcelles de $2500 \mathrm{~m}^{2}$ ont été disposées en raison de 9 parcelles par localité et tous les individus ligneux de plus de $2,5 \mathrm{~cm}$ de diamètre qui s'y trouvaient, ont été également identifiés et comptés. Suite à cela, une enquête a été menée auprès des propriétaires des exploitations agricoles (73 hommes et 35 femmes). Elle a porté sur les processus etles activités associées à la mise en place des parcelles agricoles.

Dépouillementdes données. Pratiques associées à la mise en place des parcelles agricoles. La connaissance de la pratique culturale estimportante dans la mesure où elle permet de comprendre la composition de la flore dans les agrosystèmes. Ainsi, pour une meilleure appréciation desactivités associées à la mise en place desparcelles agricoles, la manière dont les paysans procèdent a été 


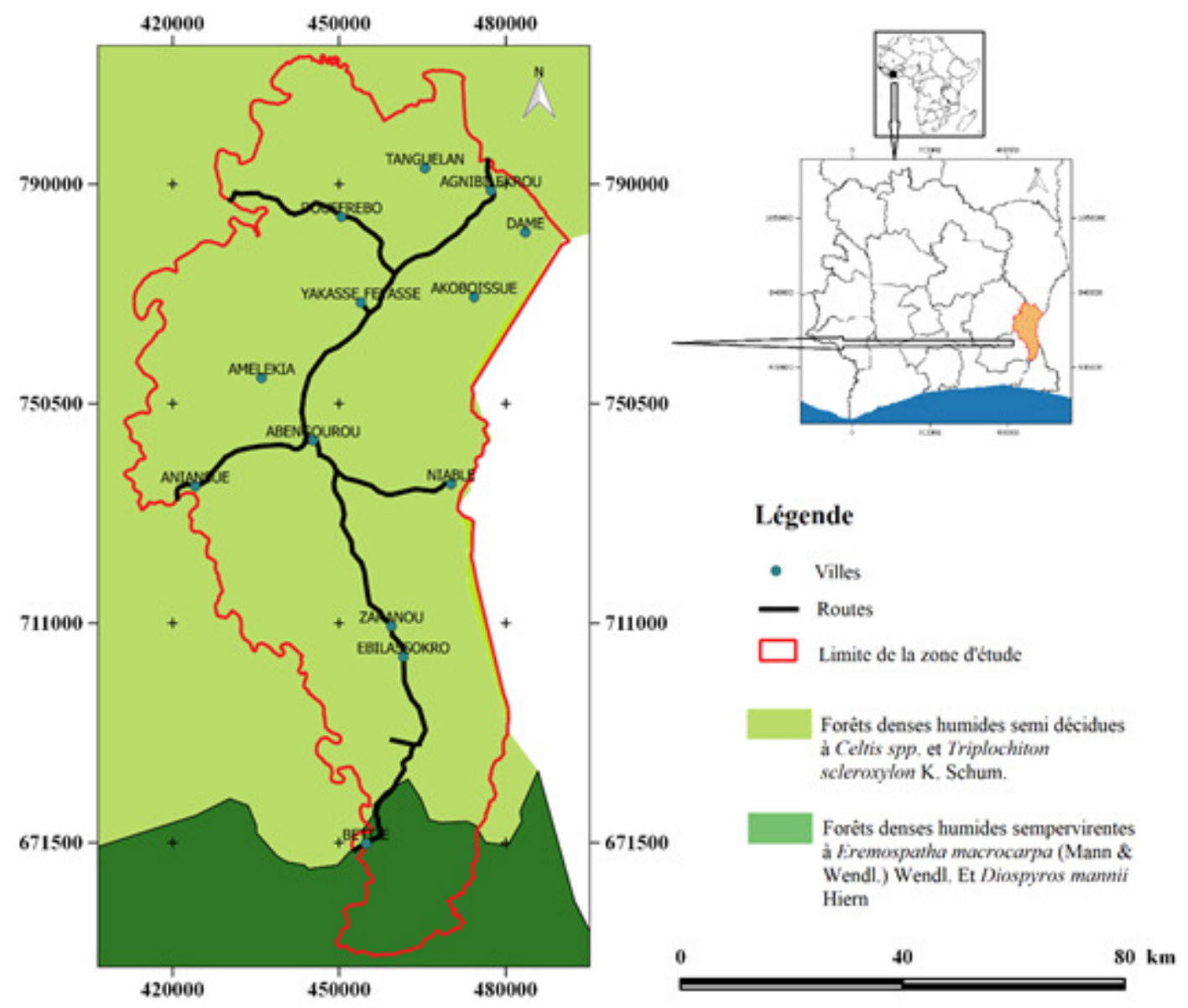

Figure 1. Localisation de la zone d'étude.

illustrée suivant les informations qu'ils ont fourni.Puis, les pourcentages des répondantsont été calculés par catégories d'activités et utilisés pour la construction des histogrammes. Ceci a permisde déterminer les périodes clés des différentes activités.

Gestion des ligneux compagnes dans les exploitations agricoles. Pour une meilleure appréciation de la gestion des ligneux compagnes dans les exploitations agricoles ciblées, l'analyse a été effectuée à trois niveaux. Premièrement, la densité et l'aire basale (Equation 1) (Monssou et al., 2016) totales des ligneux compagnes ont été calculées suivant le type et la classe d'âge des agrosystèmes. Ensuite, cette densité a été repartie selon trois classes de diamètre à hauteur de poitrine des arbres (dbh) telles que définies par Vroh et al. (2015). Il s'agit des recrus ligneux $(2,5 \mathrm{~cm}<\mathrm{dbh}<5 \mathrm{~cm})$, des jeunes arbres $(5 \mathrm{~cm}<\mathrm{dbh}<10 \mathrm{~cm})$, et des arbres matures $(\mathrm{dbh}>10 \mathrm{~cm})$. Enfin, la densité totale calculée a été également repartie entre les ligneux compagnes natifs et les ligneux compagnes introduits.

$S\left(\mathrm{~cm}^{2}\right)=\Sigma\left(\pi \times D^{2} / 4\right)$

Où : $S=$ aire basale ; $D=$ diamètre à hauteur de poitrine de la tige et $\pi=3,1416$ 
Impact de la pratique culturale sur la diversité des ligneux compagnes. L'identification et le décompte des individus ligneux dans les parcelles ont permis d'apprécier la diversité ligneuse des différents agrosystèmes. Cette appréciation a été possible en utilisant la richesse spécifique, l'indice de diversité de Shannon $\left(H^{\prime}\right)$ (Equation 2) qui est accompagné de l'équitabilité de Pielou (E) (Equation 3), le guilde de régénération et le mode de dissémination des espèces. Les données relatives à ces deux derniers paramètres proviennent des travaux de plusieurs auteurs (Feer, 1989, Hawthorne, 1996, Doucet, 2003, N'Dja,2006 et Beina,2011). La richesse spécifique renseigne sur le nombre d'espèces d'un milieu donné tandis que l'indice de Shannon (1948) et l'équitabilité de Pielou permettent d'apprécier la répartition des individus ligneux entre les espèces.

$H^{\prime}=-\sum_{i=1}^{S}\left(n_{i} / N\right) \log _{2}\left(n_{i} / N\right)$

$E=\frac{H^{\prime}}{\log _{2} s}$

Où : $H^{\prime}=$ indice de diversité de Shannon, $E=$ indice d'équitabilité, $n_{i}=$ nombre d'individus de l'espèce i, $N=$ somme du nombre d'individus de toutes les espèces, $S=$ nombre total d'espèces, $\ln S=$ valeur théorique de la diversité maximale pouvant être atteinte. L'indice d'équitabilité (E), tend vers 1 lorsque les espèces ont une répartition régulière. Il est proche ou égal à 0 lorsque les espèces ont une répartition irrégulière (Koffi et al., 2015).

Analyse statistique des données. Les données ont été analysées en utilisant le logiciel $\mathrm{R}$ (version 3.6.1) développé par $\mathrm{R}$ Core Team (2019). La densité et les paramètres de diversité (riche spécifique, indice de Shannon et équitabilité de Pielou) ont été comparés au sein des exploitations agricoles et entre les différents agrosystèmes. Pour ce faire, des analyses de variance à un facteur (ANOVA) ont été effectuées après vérification de la normalité et de l'homogénéité des variables. En présence de différence significative (test ANOVA 1) entre les moyennes pour un paramètre donné, le test de Tukey a été immédiatement appliqué au seuil de 5\%. Ce dernier permet de classer et de savoir lesquels des groupes sont différents (Ouattara et al., 2013). Dans le cas d'une absence de normalité et/ou d'homogénéité des variables, des tests non paramétriques de Kruskal-Wallis ont été appliqués.

\section{RESULTATS}

\section{Pratiques associées à la mise en place des parcelles agricoles}

Processus d'installation des parcelles. Dans la région de l'Indénié-Djuablin, la création d'une exploitation cacaoyère, commence d'abord par un défrichage de la parcelle à exploiter suivi de brulis (Fig. 2). Ensuite se déroulent les activités de dessouchages et de mise en place des buttes d'ignames sur la parcelle (Fig. 2). A ces buttes sont associés les plants ou graines de cacaoyers et certaines plantes alimentaires comme le taro (Xanthosoma sagittifolium (L.) Schott.), le bananier (Musa spp.), le manioc (Manihot esculenta Crantz), le piment (Capsicum annuит L.), la tomate (Solanum lycopersycum L.), les aubergines (Solanum spp.), le gombo (Abelmoschus esculentus (L.) Moench) et divers cucurbites. Cet ensemble aboutit quelques années plus tard à une jeune exploitation cacaoyère et ce, après des sarclages réguliers (Fig. 2). Le passage de cette jeune exploitation à une exploitation cacaoyère mature nécessite généralement des activités de sarclage, d'élagage et de réduction de la densité des espèces arborescentes autres que les plants de culture. Les pratiques observées lors de la création des exploitations cacaoyères sont les mêmes pour la création d'une exploitation 

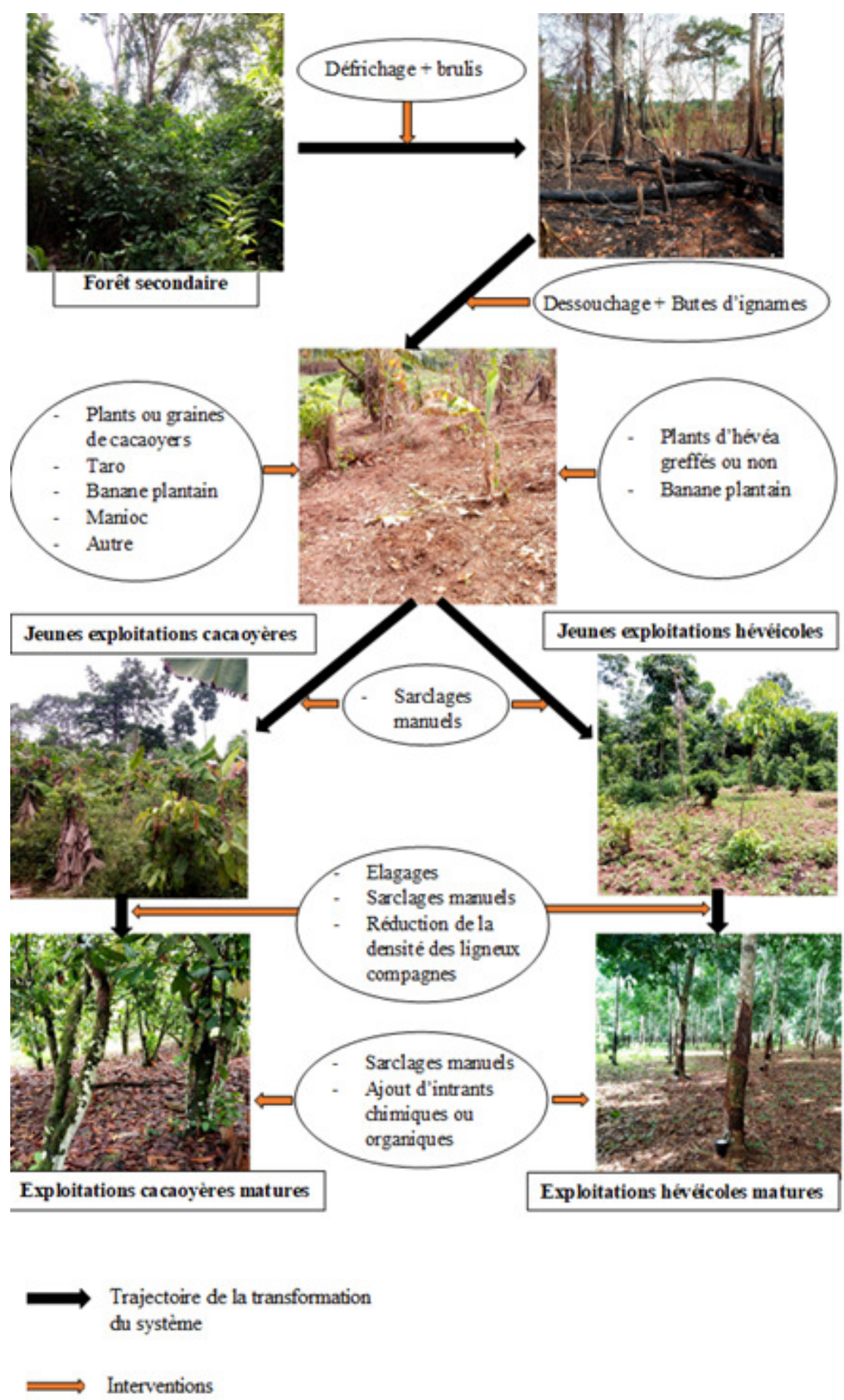

Figure 2. Processus d'installation et du maintien des exploitations cacaoyères et hévéicoles dans la région de l'Indénié-Djuablin. 
hévéicole. La seule différence est qu'en lieu et place des graines ou plants de cacaoyers, ce sont des plants de hévéa qui sont associés aux buttes d'ignames (Fig. 2).

Périodes des activités associées à la mise en place des parcelles agricoles. Les activités de défrichage et de brulis s'effectuent entre les mois de janvier et de mars où les précipitations se situent entre 16.73 et 123.84 mm (Fig. 3a). Elles sont plus réalisées en janvier et février. De mars à juin où les précipitations augmentent de 123.84 à 255.06 $\mathrm{mm}$, se déroulent les activités de dessouchages et de mise en place des buttes d'ignames (Fig. 3b). Ceci s'effectue préférentiellement au cours des mois de mars et avril. Quant aux activités de sarclage, elles commencent en novembre et prennent fin dans le mois de mai de l'année suivante (Fig. 3c). Les activités de ce genre sont fréquentes dans les mois de janvier et de février où les précipitations sont respectivement de $16.73 \mathrm{~mm}$ et de $60.10 \mathrm{~mm}$. Toutefois, la fréquence de sarclage annuel est au maximum de quatre sarclages dans les exploitations de moins de 5 ans, de trois sarclages dans les exploitations de 5 à 15 ans et deux sarclages dans celles âgées de plus de 15 ans (Fig. 3d).

Gestion des ligneux compagnes dans les exploitations agricoles. La densité totale des espèces arborescentes dans les parcelles de forêts en reconstitution est en moyenne de 619,33 tiges par ha avec une aire basale moyenne de $74,26 \mathrm{~m}^{2}$ par ha (Fig. 4a). Elle est comprise entre 27,66et 49,33 tiges par ha dans les exploitations cacaoyères où l'aire basale moyenne se situe entre 2,58 et $8,99 \mathrm{~m}^{2}$ par ha (Fig. 4a). Dans les exploitations hévéicoles où l'aire basale se situe entre 0,08 et $0,48 \mathrm{~m}^{2}$ par ha, la densité moyenne des espèces arborescentes est comprise entre 1,33 et 7,00 tiges par ha (Fig. 4a). On note une baisse significative $\left(x^{2}=63,39 ; \mathrm{P}<0,001\right)$ de la densité des espèces arborescentes des forêts en reconstitution après installation des exploitations cacaoyères et hévéicoles. Cette baisse de la densité est fortement et positivement corrélée $(r=0,9986 ; \mathrm{P}<0,001)$ à l'aire basale selon le test de corrélation de Person.

Suivant le diamètre à hauteur de poitrine (dbh) des espèces arborescentes, la densité moyenne des individus ligneux ayant un dbh compris entre 2,5 et $5 \mathrm{~cm}, 5$ et $10 \mathrm{~cm}$ et un dbh de plus de $10 \mathrm{~cm}$ est respectivement de 190, 174,33 et 255 tiges par ha dans les forêts en reconstitution (Fig. 4b). Dans les exploitations cacaoyères et hévéicoles, seuls les individus de plus de $10 \mathrm{~cm}$ de diamètre persistent véritablement. Leur densité est de 41,00 tiges par ha dans les exploitations cacaoyères de moins de 5 ans, de 25,67 tiges par ha dans celles de 5 à 15 ans et de 49,33 tiges par ha dans les exploitations cacaoyères de plus de 15 ans (Fig. 4b). Au niveau des exploitations hévéicoles, cette densité se situe entre 1,33 et 3,33 tiges par ha (Fig. 4b). Les différences observées entre la densité des classes de diamètre sont significatives (dbh $(2,5-5 \mathrm{~cm}): x^{2}=77.35 ; \mathrm{P}<0,001 ; \mathrm{dbh}(5-10$ cm) : $x^{2}=63.45 ; \mathrm{P}<0,001 ; \mathrm{dbh}(\mathrm{e} " 10 \mathrm{~cm})$ : $\left.x^{2}=63.21 ; \mathrm{P}<0,001\right)$ et baissent d'un milieu à un autre.

De 41 tiges/ha dans les exploitations cacaoyères de moins de 5 ans, la densité des espèces forestières passe à 16,33 tiges par ha dans celles de plus de 15 ans (Fig. 4c). Contrairement à cela, la densité des espèces exotiques est de 3,6 et 33 tiges par ha respectivement dans les exploitations cacaoyères de moins de 5 ans, de 5 à 15 ans et de plus de 15 ans. Dans les exploitations hévéicoles, seules les espèces forestières persistent avec une densité de 3,33, 7 et 1,33 tiges par ha respectivement dans les exploitations de moins de 5 ans, de 5 à 15 ans et plus de 15 ans (Fig. 4c).

\section{Impact de la pratique culturale sur la diversité des ligneux compagnes}

Richesse et composition floristique. Au total 109 espèces arborescentes réparties en 60 genres et 35 familles ont été recensées dans 

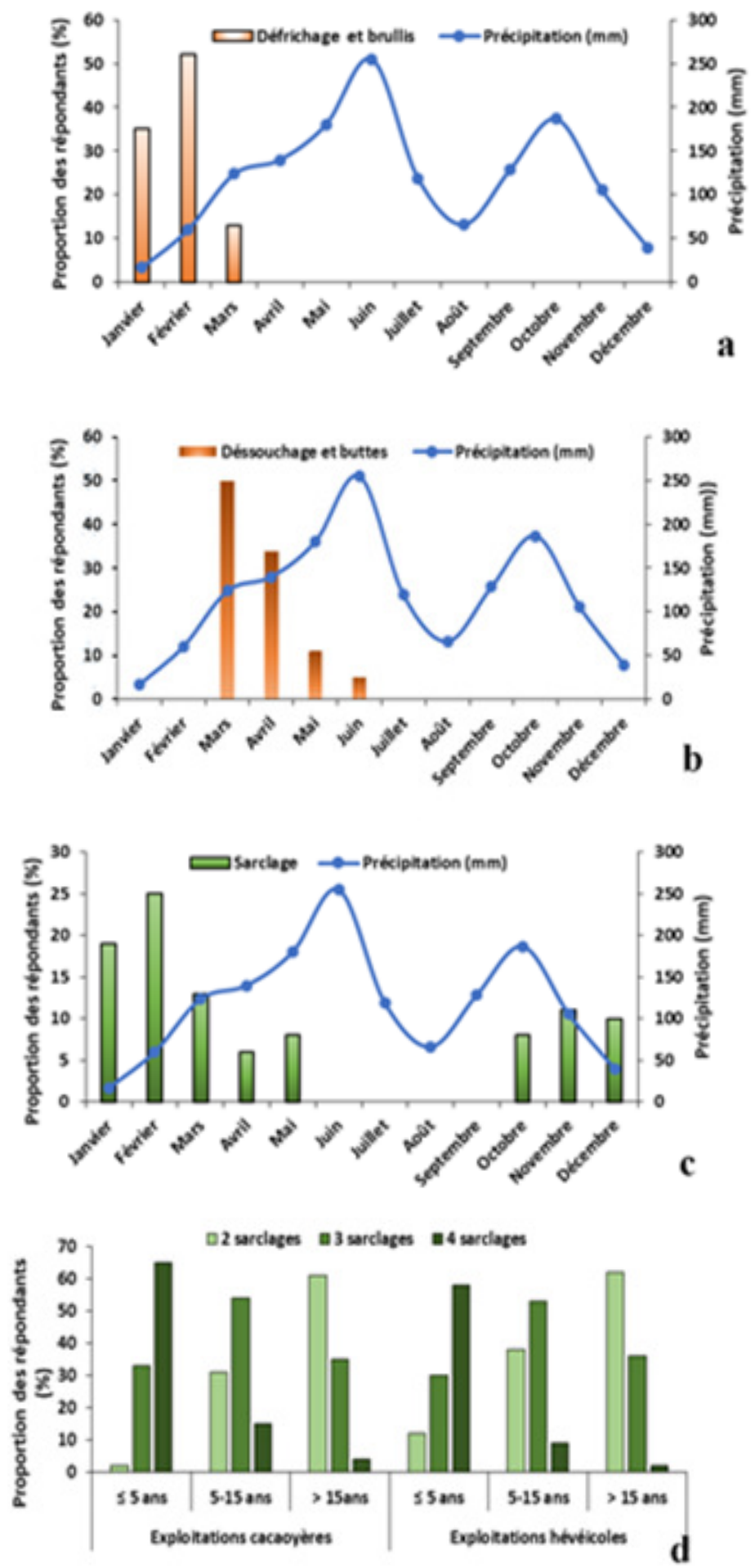

Figure 3. Périodicité des pratiques liées à la création des exploitations agricoles, a) période de défrichage et de brulis ; b) période de dessouchage et de mise en place des butes; c) période de sarclage ; d) fréquence de sarclage. 
Ligneux a usages multiples dans les exploitations agricoles
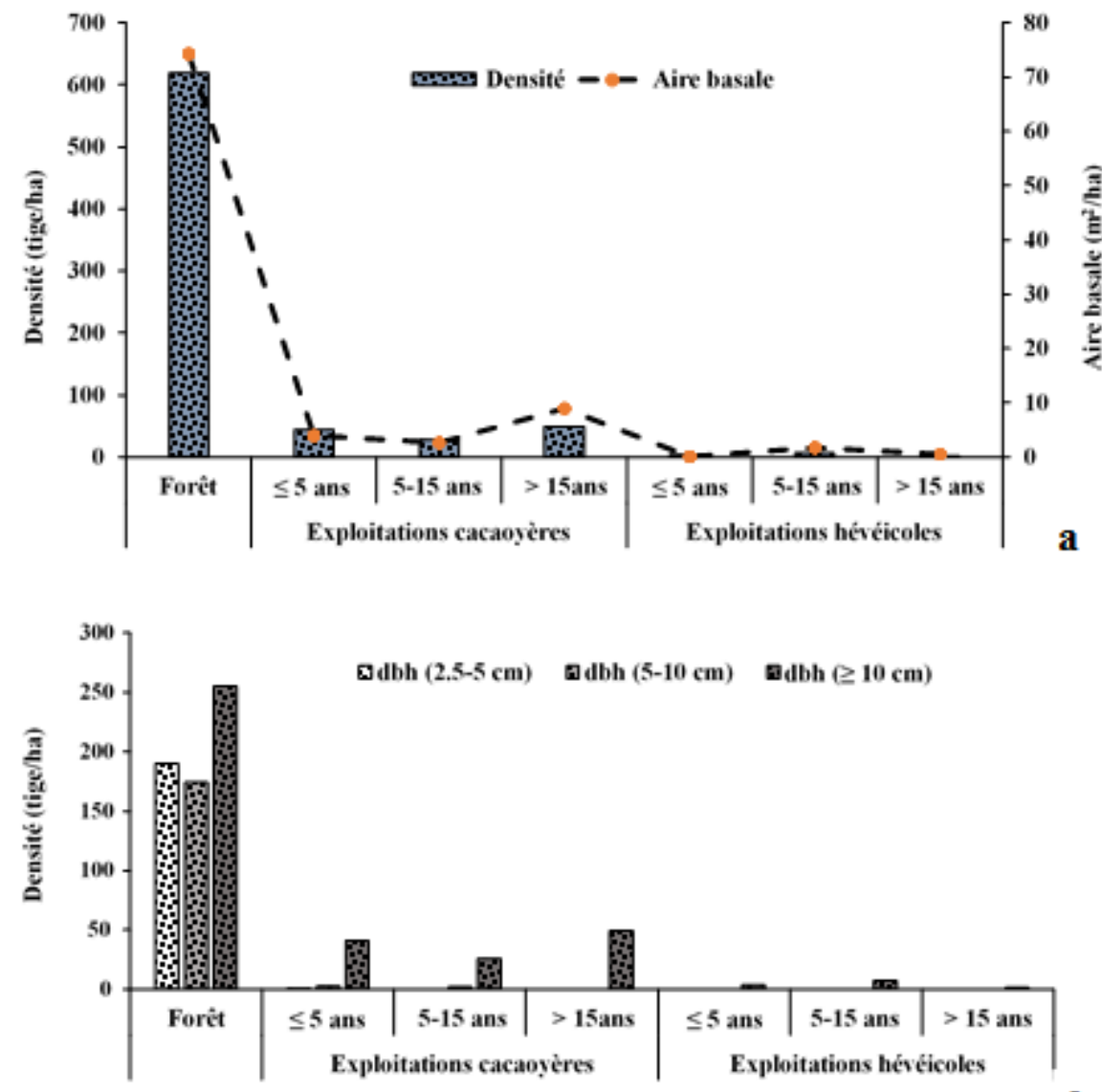

b

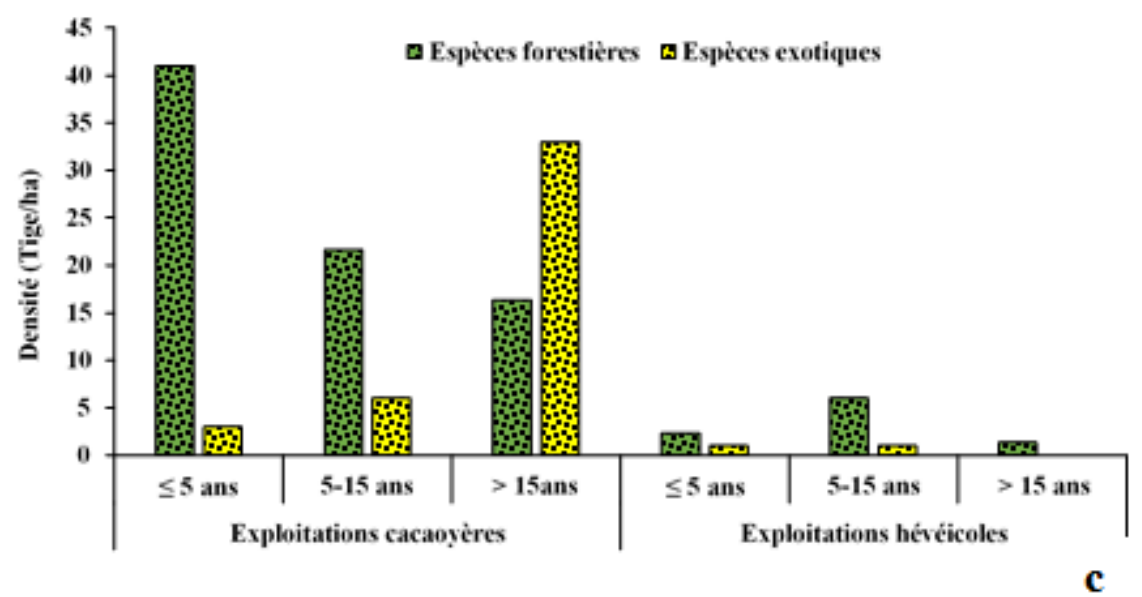

Figure 4. Gestion des espèces arborescentes dans les agrosystèmes a) Densité et aire basale totale des espèces arborescentes; b) Répartition des individus ligneux par classe de diamètres; c) Evolution de la densité des espèces forestières et des espèces exotiques suivant la classe d'âge des exploitations cacaoyères et hévéicoles. $<5$ ans : exploitations agricoles de moins de moins de 5 ans, 5-15 ans : exploitations agricoles de 5 à 15 ans, $>15$ ans : exploitations agricoles de plus de 15 ans. dbh (2.5-5 $\mathrm{cm})$ : diamètre à hauteur de poitrine compris entre 2.5 et $5 \mathrm{~cm}$, dbh $(5-10 \mathrm{~cm})$ : diamètre à hauteur de poitrine compris entre 5 et $10 \mathrm{~cm}, \mathrm{dbh}(>10 \mathrm{~cm})$ : diamètre à hauteur de poitrine de plus de $10 \mathrm{~cm}$. 
les forêts en reconstitutions. Dans ces milieux, 9 espèces ont été rencontrées dans plus des $50 \%$ des relevés (Tableau 1). Il s'agit entre autres de Scottellia klaineana, Entandrophragma angolense, Triplochiton scleroxylon. Ces espèces qui pour la plupart sont zoochores ou anémochores se répartissent entre deux guildes de régénération à savoir : les espèces pionnières et les espèces héliophiles non pionnières. Les familles ayant un grand nombre d'espèces dans ces forêts sont les Fabaceae (19 espèces), les Malvaceae (12 espèces), les Rubiaceae (12 espèces) et les Moraceae (8 espèces) (Fig. 5a). Dans les exploitations cacaoyères, il a été recensé 63 espèces réparties en 49 genres et 25 familles. Seules Entandrophragma angolense etPersea americana ont été rencontrées dans plus de $50 \%$ des relevés (Tableau 1). Toutefois les ligneux généralement épargnés dans ces exploitations agricoles sont anémochores et appartiennent aussi à deux guildes de régénération comme c'est le cas des forêts. Les Fabaceae (15 espèces), les Malvaceae (9

TABLEAU 1. Guilde de régénération, diaspore et mode dissémination des diaspores des espèces arborescente fréquemment rencontrées dans les forêts en reconstitution et les exploitations cacaoyères et hévéicoles

\begin{tabular}{|c|c|c|c|c|}
\hline Espèces arborescentes & Fréquence & $\begin{array}{l}\text { Guilde de } \\
\text { régénération }\end{array}$ & Diaspore & $\begin{array}{l}\text { Mode de } \\
\text { dissémination }\end{array}$ \\
\hline
\end{tabular}

\section{Forêts en reconstitution}

Scottellia klaineana

Ficus mucuso

Entandrophragma angolense

Ceiba pentandra

Cola gigantea

Dracaena arborea

Antrocaryon micraster

Pterygota macrocarpa

Triplochiton scleroxylon

Albizia adianthifolia

Vitex fosteri

Albizia zygia

\section{Exploitations cacaoyères}

Entandrophragma angolense

Persea americana

Ceiba pentandra

Trema orientalis

Albizia adianthifolia

Mansonia altissima

77.78
72.22
72.22
66.67
66.67
66.67
61.11
55.56
50
44.44
27.78
27.78

$\mathrm{SB}$
$\mathrm{P}$
$\mathrm{Np}$
$\mathrm{P}$
$\mathrm{N}$
$\mathrm{P}$
$\mathrm{N}$
$\mathrm{Np}$
$\mathrm{P}$
$\mathrm{N}$
$\mathrm{N}$
$\mathrm{N}$

SB

$\mathrm{P}$

$\mathrm{Np}$

$\mathrm{P}$

$\mathrm{Np}$

$\mathrm{P}$

$\mathrm{Np}$

$\mathrm{Np}$

$\mathrm{P}$

$\mathrm{Np}$

$\mathrm{Np}$

$\mathrm{Np}$

70.37

51.85

48.15

40.74

33.33

25.93

Exploitations hévéicoles

Ceiba pentandra

Spathodea campanulata

Bombax buonopozense

Persea americana

\begin{tabular}{|c|c|c|c|}
\hline 70.37 & $\mathrm{~Np}$ & Ptérochore & Anémochorie \\
\hline 51.85 & I & Sarcochore & Zoochorie \\
\hline 48.15 & $\mathrm{P}$ & Pogonochore & Anémochorie \\
\hline 40.74 & $\mathrm{P}$ & Sarcochore & Zoochorie \\
\hline 33.33 & $\mathrm{~Np}$ & Ptérochore & Anémochorie \\
\hline 25.93 & $\mathrm{~Np}$ & Ptérochore & Anémochorie \\
\hline 37.04 & $\mathrm{P}$ & Pogonochore & Anémochorie \\
\hline 14.81 & $\mathrm{P}$ & Ptérochore & Anémochorie \\
\hline 11.11 & $\mathrm{P}$ & Pogonochore & Anémochorie \\
\hline 11.11 & I & Sarcochore & Zoochorie \\
\hline
\end{tabular}

$\mathrm{P}=$ Pionnière, $\mathrm{Np}=$ héliophiles non pionnières, $\mathrm{SB}=$ Sous-bois

Zoochorie

Zoochorie

Anémochorie

Anémochorie

Zoochorie

Zoochorie

Autochorie

Zoochorie

Anémochorie

Anémochorie

Zoochorie

Anémochorie

Ptérochore 


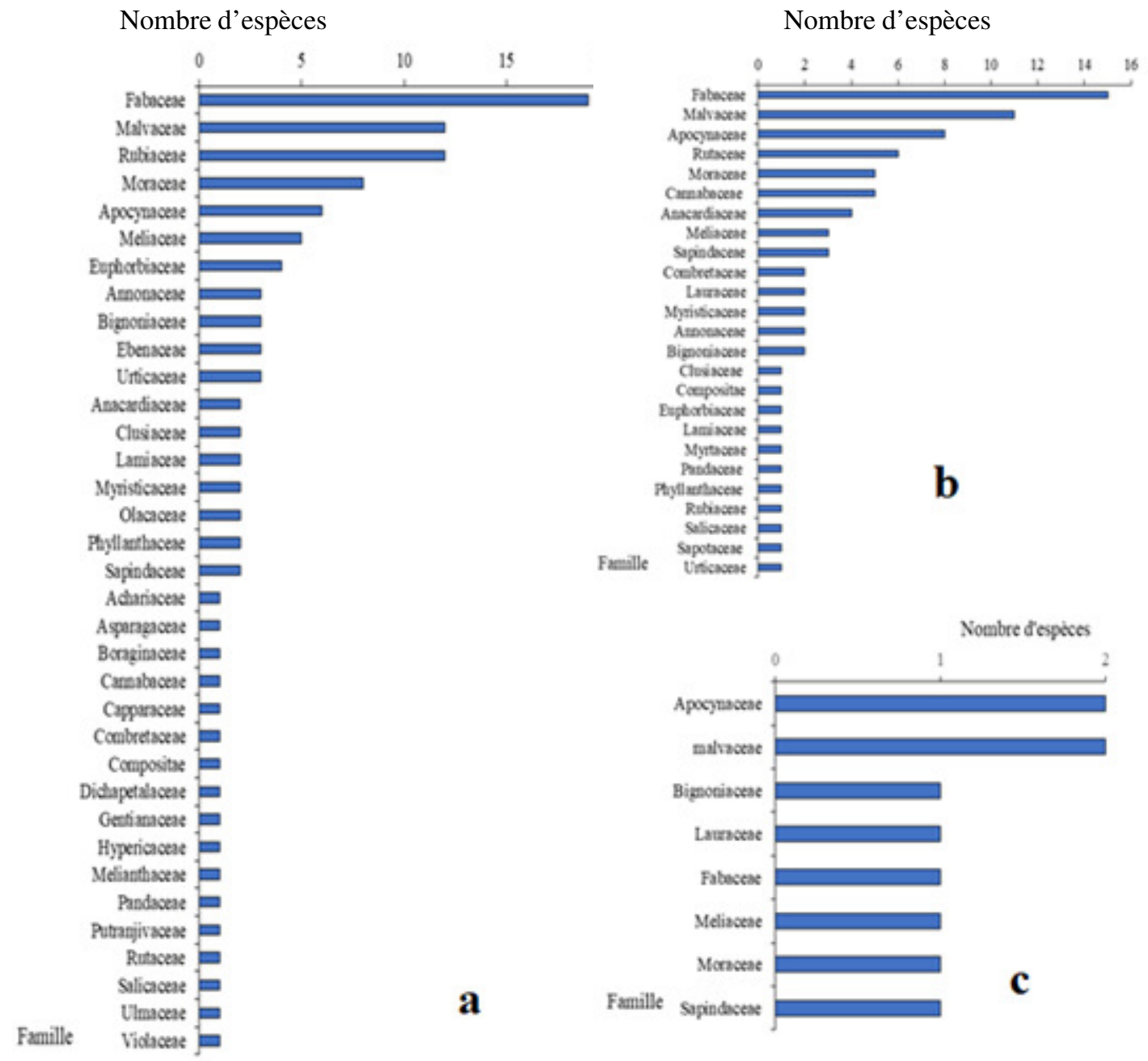

Figure 5. Spectre des familles des espèces arborescentes présentent dans a) les forêts en reconstitutions ; b) les exploitations cacaoyères ; c) les exploitations hévéicoles

espèces) et les Apocynaceae (8 espèces) sont les familles les plus riches en espèces dans ces exploitations agricoles (Fig. 5b). Concernant le nombre total d'espèces arborescentes recensées dans les exploitations hévéicoles, il est de 10 espèces réparties en 10 genres et 8 familles avec un maximum de deux espèces par familles (Fig. $5 \mathrm{c}$ ). Toutes les espèces inventoriées dans ce type d'exploitation agricole ont une fréquence de moins de 50\% (Tableau 1). De plus, elles sont pour la plupart anémochores et pionnières.
Diversité. Le nombre moyen d'espèces arborescentes est de 15.5 espèces dans les parcelles de forêts en reconstitution (Tableau 2). Dans les exploitations cacaoyères, c'est en moyenne 4.92,4.4 et 6.17 espèces de ligneux compagnes qui ont été recensées respectivement dans les exploitations de moins de 5 ans, de 5 à 15 ans et de plus de 15 ans (Tableau 2). Au niveau des exploitations hévéicoles, le nombre moyen d'espèces de ligneux compagnes recensées se situe entre 2.08 et 2.5 espèces (Tableau 2). Ces valeurs 
moyennes diffèrent significativement d'un milieu à un autre $(\mathrm{F}=48,22 ; \mathrm{P}<0,001)$. Selon le test de Tukey, les forêts en reconstitutions sont plus riches en espèces arborescentes que les exploitations cacaoyères et hévéicoles. Par ailleurs, le nombre moyen d'espèces arborescentes recensées dans les exploitations cacaoyères augmente significativement avec la classe d'âge tandis qu'il reste relativement faible dans les exploitations hévéicoles. Tout comme les valeurs moyennes d'espèces, celles de l'indice de diversité de Shannon ( $\left.\mathrm{H}^{\prime}\right)$ varient et diffèrent significativement $(\mathrm{F}=20,28$; $\mathrm{P}<0.001)$ d'un milieu à un autre (Tableau 2). Les forêts en reconstitution enregistrent les plus grandes valeurs d'indice de diversité $\left(H^{\prime}=2.68\right)$ et celles des exploitations hévéicoles les plus faibles valeurs qui se situent entre 0.69 et 0.86 bits/individu. Cependant, entre ces deux extrêmes se situent les exploitations cacaoyères dont les valeurs moyennes d'indice de diversité varient entre 1.06 et 1.35. Notons qu'au sein des exploitations cacaoyères, l'indice de diversité est faible dans les exploitations de 5 à 15 ans $\left(\mathrm{H}^{\prime}=1.06\right)$ et élevé dans celles de moins de 5 ans $\left(H^{\prime}=1.34\right)$ et de plus de 15 ans $\left(H^{\prime}=1.35\right)$.

\section{DISCUSSION}

Pratiques associées à la mise en place des parcelles agricoles. Dans la présentezone d'étude, les paysans procèdent pratiquement de la même manière pour l'installation et le maintien des exploitations cacaoyère ou hévéicoles. Particulièrement, ils défrichent et brûlent d'abord les débris végétaux dans les parcelles à exploiter. Ces activités de préparation s'effectuent généralement en saison sèche (de janvier à février) où les conditions de mise à feu des débris végétaux dans les parcelles sont plus appropriées. Cette technique est d'ailleurs reconnue comme une pratique ancestrale courante en agriculture (Sow et al., 2013; Van Wilgen et al., 2014). Elle permet aux paysans de dégager plus d'espace afin d'exploiter au mieux la parcelle. Ainsi, après avoir brûlé la biomasse végétale 
morte, dans la parcelle, les paysans procèdent aux dessouchages. Cette activité couramment appelée "pâme" dans le jargon des paysans, consiste à déraciner les restes de racines d'arbres et d'arbustes afin de faciliter la mise en place des butes dans la parcelle. Notons qu'aux butes sont associés les plants de cultures et certaines plantes alimentaires telles que l'igname (Dioscorea sp.), le taro (Xanthosoma sagittifolium), le bananier (Musa spp), le manioc (Manihot esculenta) et le piment (Capsicum annuum). Ceci permet aux paysans d'avoir des moyens de subsistance en attendant que la plantation entre en production. Cette observation a été aussi faite par Somboonsuke et al. (2011) en Thaïlande. Selon ces auteurs, les paysans thaïlandais associent des cultures annuelles aux plants de Hevea brasiliensis Müll. Arg afin de soutenir leur revenu familial durant les deux premières années qui précède la production de la plantation. Toutefois, comme le relève Kpangui et al. (2015), contrairement aux autres plantes alimentaires, le bananier (Musa sp.) est généralement utilisé pour faire de l'ombrage aux jeunes plants de cultures. Dans ces jeunes exploitations, la fréquence de sarclage élevée serait une stratégie pouréviter l'enherbement des parcelles qui constitue un obstacle au développement des plants de cultures. La baisse de cette fréquence avec l'âge de la plantation serait liée au fait qu'un processus naturel de lutte contre l'enherbement est opéré par l'effet de la canopée (Ipou et al., 2011). En outre, au cours de l'évolution des plants de cultures, des activités d'élagage sont entreprises et les arbres devenus inutiles ou gênants du fait de leur nombre, sont éliminés.

Gestion des ligneux compagnes dans les exploitations agricoles. Au cours de l'installation et du maintien des nouvelles parcelles agricoles dans la présentezone d'étude, la densité et l'aire basale des espèces arborescentes associées aux plants de cultures régressent significativement. Cette situation est due au fait que durant les activités de défrichage des parcelles, les paysans éliminent considérablement les recrus ligneux, les jeunes arbres et près de $80 \%$ des arbres matures. Les arbres matures généralement épargnés sont Entandrophragma angolense (Welw.) C. DC., Ceiba pentandra (L.) Gaertn., Albizia adianthifolia (Schumach.) W. Wight, Mansonia altissima (A.Chev.) A.Chev. et Trema orientalis (L.) Blume. Dans les exploitations cacaoyères, ces arbres sont épargnés parce que les paysans les considèrent comme compatibles avec la croissance et la production du cacaoyer (Adou Yao et al., 2016). Leur présence dans ces exploitations agricoles pourrait être aussi liée au fait que ces espèces ont atteint un diamètre difficile à abattre. Cependant, avec l'âge des plantations, des arbres fruitiers tels que Persea americana Mill., Cola nitida (Vent.) Schott \& Endl. et Garcinia kola Heckel, sont intentionnellement introduits tandis que certains arbres natifs sont éliminés pour la croissance des cacaoyers. C'est ce qui explique en partie la légère variation de la densité et de l'aire basale des espèces arborescentes dans ces exploitations agricoles. Dans les exploitations hévéicoles, par contre, les valeurs de la densité et de l'aire basale des espèces arborescentes sont relativement faibles. Cette situation résulte du fait que seuls les individus matures de Ceiba pentandra y sont généralement épargnés, car le pied mort de cette espèce d'arbre est perçu par les paysans comme l'hôte potentielle du champignon Fomes lignosus (Thierry, 2005).

Il est évident que cette forme d'installation et de maintien des nouvelles parcelles agricoles n'est pas sans conséquence sur la diversité des espèces arborescentes dans notre zone d'étude.

Impact de la pratique culturale sur la diversité des ligneux compagnes. Les investigations dans le paysage agricole de la présentezone d'étude ont montré que 42,20\% des espèces arborescentes inventoriées dans les forêts en reconstitution ont été éliminées dans les exploitations cacaoyères contre 
90,82\% dans les exploitations hévéicoles. Ceci serait en partie dû au fait que ces espèces sont perçues par les paysans comme étant nuisibles aux plants de cultures. De telles observations ont été faites par Adou Yao et al. (2016) au centre de la Côte d'Ivoire. Selon eux, certaines espèces arborescentes sont éliminées dans les plantations parce qu'elles sont vues par les paysans comme espèces asséchant les sols ou espèces à influence négative sur le développement des cacaoyers. Il s'agit pour la plupart de Triplochiton scleroxylon K. Schum., Cola gigantea var. glabrescens Brenan \& Keay, Albizia zygia (DC.) J. F. Macbr., Celtis zenkeri Engl. et de Ficus exasperata Vahl.

Malgré la réduction drastique de cette catégorie d'espèces arborescentes, les exploitations cacaoyères de la présentezone d'étude présentent une structure plus ou moins similaire à celle des forêts en reconstitution, en termes de nombre d'espèces par famille. Ceci n'est pas le cas des exploitations hévéicoles. Toutefois, la dominance des Fabaceae dans les exploitations cacaoyères et les forêts en reconstitution est due au fait que les paysans épargnent généralement certains individus ligneux appartenant à cette famille pour assurer la fertilité du sol. Cela résulterait des campagnes de vulgarisation des techniques d'enrichissement des sols à partir des légumineuses arborées fixatrices d'azotes (Gnahoua et al., 2008). Les variations significatives du nombre moyen d'espèces et de l'indice de diversité observées dans les exploitations cacaoyères, sont en partie dues au fait que les paysans y introduisent volontairement de nouvelles espèces de ligneux compagnes. Dans les exploitations hévéicoles, par contre, ces paramètres sont relativement constants et faibles du fait que les paysans n'y accordent pas assez d'importance aux espèces arborescentes autres que les plants de cultures. C'est d'ailleurs ce qui explique le fait que les exploitations cacaoyères soient plus diversifiées en espèces arborescentes que les exploitations hévéicoles dans la présentezone d'étude. Partant de ce constat, les exploitations cacaoyères pourraient être qualifiées de système agroforestier pluristratifié et les exploitations hévéicoles, de système monoculture. En effet, les systèmes agroforestiers sont plus diversifiés que systèmes monocultures (Gillison et al., 2004). En outre, ceci pourrait affecter le processus de reconstitution de la forêt après abandon de ces exploitations agricoles. En effet, la diversité d'espèces, de guilde de régénération et de mode de dissémination des diaspores déterminent la dynamique de la reconstitution post-culturale (Condit et al., 2000).

Parlant de reconstitution post-culturale, les forêts en reconstitution caractérisées par un retour remarquable d'espèces forestières sont celles qui enregistrent de fortes présences d'espèces zoochores et anémochores.Cela serait dû à l'action de deux facteurs additionnels; à savoir (i) la présence remarquable des semenciers zoochores (Scottellia klaineana, Cola gigantea) et anémochores (Entandrophragma angolense, Triplochiton scleroxylon); and (ii) la présence des mammifères, des oiseaux et du vent, vecteurs potentiels de cesmodes de dissémination. En effet, selon N'Dja (2006), les modes de dissémination combinés, avec la présence des semenciers dans un rayon compatible avec la puissance du vecteur de la dissémination, influencent les capacités d'apparition d'une espèce végétale dans un site.Cependant, la forte présence d'espèces héliophiles non pionnières montre que les forêts dites en reconstitution sont au deuxièmestade de la succession post-culturale (Khan, 1982).Toutefois, tout ceci dénote un bon fonctionnement de ces milieux. En revanche, dans les exploitations cacaoyères et hévéicoles de la présente zone d'étude, l'abattage sélectif des ligneux compagnes pourrait plus ou moins ralentir le processus de la régénération postculturale. Cela se justifie par le fait que les paysans réduisent l'action des mammifères et des oiseaux en n'épargnant que des espèces 
anémochore. Pourtant contrairement au vent, les mammifères et les oiseaux sont très actifs dans la dispersion des graines et la dynamique de la reconstitution post-culturale (Chatelain et al., 2001; Ganesh et Davidar, 2001). Par ailleurs, les exploitations cacaoyères auraient un fort potentiel de reconstitution de la diversité floristique comparé aux exploitations hévéicoles. En effet, les espèces de ligneux compagnes généralement épargnées dans les exploitations hévéicoles sont caractéristiques du premier stade de la succession postculturale tandis que celles épargnées dans les exploitations cacaoyères caractérisent les deux premiers stades. Notons que la faible présence d'espèces zoochores dans les exploitations cacaoyères serait une stratégie mise en place par les paysans pour lutter contre les ravageurs de culture tels que les rongeurs.

\section{CONCLUSION}

Cette étude a montré que dans la région de l'Indénié-Djuablin située à l'Est de la Côte d'Ioire, les paysans procèdent à un abattage quasi-total des défriches de la forêt primaire ou secondaire suivi de brûlis pour installerdes exploitations agricoles. Ils réduisent considérablement la densité des recrus ligneux, des jeunes arbres et des arbres matures. Par ailleurs, ces paysans ont toujours recours à l'ancienne pratique associant culture pérenne et autres ligneux compagnes. Dans les exploitations cacaoyères, cette pratique s'étend tout au long des stades de développement des plants de cultures tandis que dans les exploitations hévéicolescela se limite aux années qui précèdent la production des plants de cultures. Contrairement aux exploitations hévéicoles, des espèces arborescentes sont épargnées et introduites dans les exploitations cacaoyères pour divers usages. Ceci garantit la conservation d'un nombre important d'espèces arborescentes dans ces exploitations agricoles ce qui n'est pas le cas dans les exploitations hévéicoles. Suivant ces observations, les exploitations cacaoyères de zone d'étude peuvent être qualifiées de systèmes agroforestiers pluristratifiés, conservatrices de la biodiversité locale et capables de restaurer la diversité floristique. A l'inverse, les exploitations hévéicoles peuvent être qualifiées de systèmes plein soleil qui maximisent la production.

\section{REFFERENCES BIBLIOGRAPHIQUES}

Adou Yao, C.Y., Kpangui, K.B., Vroh, B.T.A. et Ouattara, D. 2016. Pratiques culturales, valeurs d'usage et perception des paysans des espèces compagnes du cacaoyer dans des agroforêts traditionnelles au centre de la Côte d'Ivoire. Revue d'ethnoécologie 9: 1-17.

Asigbaase, M., Sjogersten, S., Lomax, B.H. and Dawoe, E. 2019. Tree diversity and its ecological importance value in organic and conventional cocoa agroforests in Ghana. PLoS ONE 14(1):1-19. DOI: 10.1371/journal.pone.0210557

Beina, D. 2011. Diversité floristique de la forêt dense semi-décidue de Mbaïki, république centrafricaine : étude expérimentale de l'impact de deux types d'intervention sylvicole. Thèse de doctorat. Université de Picardie Jules Verne, France. 226pp.

Bhagwat, S.A, Willis, K.J., Birks, H.J.B. and Whittaker, R.J. 2008. Agroforestry: A refuge for tropical biodiversity? Trends in Ecology and Evolution 23:261-267.

Carrière, S.M. 2002. L'abattage sélectif : Une pratique agricole ancestrale au service de la régénération forestière. Bois et Forêts des Tropiques 272(2):45-62.

Chatelain, C., Kadjo, B., Kone, I. et Refisch, J. 2001. Relations faune - flore dans le Parc National de Taï : une étude bibliographique. Tropenbos - Côte d'Ivoire Abidjan, Côte d'Ivoire. 187pp.

Condit, R., Ashton, P.S, Baker, P. 2000. Spatial Patterns in the Distribution of Tropical Tree Species. Science 288:1414-1418.

Deheuvels, O. 2011. Compromis entre productivité et biodiversité sur ungradient 
d'intensité de gestion de systèmes agroforestiers à base de cacaoyers de Talamanca, Costa Rica Thèse. Montpellier SupAgro, France. 185pp.

Doucet, J-L. 2003. L'alliance délicate de la gestion forestière et de la biodiversité dans les forêts du centre du Gabon. Thèse de doctorat. Faculté universitaire des sciences agronomiques de gembloux, Belgique. 390pp.

Feer, F. 1989. Comparaison des régimes alimentaires de Cephalophus callipygus et C. dorsalis, Bovidés sympatriques de la fôret sempervirente africaine. Mammalia 53(4):563-604. DOI :10.1515/mamm. 53.4 .563

Folega, F., Atakpama, W., Kanda, M., Wala, K., Batawila, K. and Akpagana, K. 2019. Agroforestry parklands and carbon sequestration in tropical Sudanese region of Togo. Revue Marocaine des Sciences Agronomiques et Vétérinaires 7(4):563570.

Ganesh, T. and Davidar, P. 2001. Dispersal modes of tree species in the wet forests of southern Western Ghats. Current science 80 (3):394-399.

Gillison, A.N., Liswanti N., Budidarsono S., Van Noordwijk M., andTomich T.P. 2004. Impact of cropping methods on biodiversity in coffee agroecosystems in Sumatra, Indonesia. Ecology and Society 9(2):7. DOI: 10.5751/ES-00657-090207

Giri ,A., Kumar, G., Arya, R., Mishra, S. and Mishra, A.K. 2019. Carbon sequestration in Populus deltoides based agroforestry system in Northern India. International Journal of Chemical Studies 7(1):21842188.

Gnahoua, G.M., Kouassi, F.Y., Angui, P.K.T., Balle, P., Olivier, R. et Peltier, R. 2008. Effets des jachères à Acacia mangium, Acacia auriculiformis et Chromolaena odorata sur la fertilité du sol et les rendements de l'igname (Dioscorea spp.) en zone forestière de Côte d'Ivoire.
Agronomie Africaine 20(3):291-301. DOI: 10.4314/aga.v20i3.46269

Gockowski, J. and Sonwa, D. 2010. Cocoa intensiûcation scenarios and their predicted impact on $\mathrm{CO}_{2}$ emissions, biodiversity conservation, and rural livelihoods in the Guinea Rain Forest of West Africa. Environmental Management 48:307-321. DOI : 10.1007 / s00267-010-9602-3.

Guillaumet, J.L. et Adjanohoun, E. 1971. Le milieu naturel en Côte d'Ivoire : La végétation de la Côte d'Ivoire. Mémoires ORSTOM, Paris (50):61-261.

Hawthorne, W.D. 1996. Holes and the sums of parts in Ghanaian forest: regeneration, scale and sustainable use. Proceedings of the Royal Society of Edinburgh. Section B. Biological Sciences 104:75-176. DOI: 10.1017/S0269727000006126

Ipou, I.J., Touré., A., Adou, L.M.D., Touré, Y. and Aké, S. 2011. Influence of some cover leguminous plants on the infestation degree of cotton plant major weeds. African Journal of Agricultural Research 6(5):1097-1108. DOI : 10.5897/AJAR09. 644

Khan, F. 1982. La reconstitution de la forêt tropicale après une culture traditionnelle, (Sud oust de la Côte d'Ivoire). Paris: ORSTOM. 150pp.

Koffi, K.A.D., Adou, Yao, C.Y., Vroh, B.T.A., Gnagbo, A. et N'guéssan, K.E. 2015. Diversités floristique et structurale des espaces anciennement cultivés du Parc National d'Azagny (Sud de la Côte d'Ivoire). European Journal of Scientific Research 134(4):415-427.

Koné, M. 2015. Évolution du couvert forestier dense et impact de la déforestation sur la migration de la boucle du cacao en Côte d'Ivoire. Thèse de doctorat. Université Nangui Abrogoua, Côte d'Ivoire. 185pp.

Koné, M., Coulibaly, L., Malan, D.F., Neuba, D.F.R., Kouadio, Y.L., Tra, B.F.H. et N'guessan, K.E. 2013. Apport de la télédétection spatiale à l'étude de l'impact 
de la cacao-culture sur la déforestation dans la région de l'Indénié-Djuablin, à l'est Côte d'Ivoire. European Journal of Scientific Research 98(4):489-509.

Koné, M., Kouadio, K., Kouadio., Y.L., Neuba, D.F.R. et Malan, D.F. 2014. Dégradation de la forêt dense humide tropicale, cas de la région de l'Indénié-Djuablin à l'est de la Côte d'Ivoire. Journal of Animal \& Plant Sciences 21(3):3324-3338.

Kouakou, Y.B., Malan, D.F., Litta, A.L., Kouassi, K.G. et Bakayako A. 2018. Plantes indicatrices des terres fertiles et de changement de saisons chez les Koulango et les Lobi riverains du Parc national de la Comoé, Nord-Est de la Côte d'Ivoire. Afrique SCIENCE 14(6):234-246.

Kpangui, K.B., Vroh, B.T.A., Goné, B.Z.B. et Adou Yao, C.Y. 2015. Diversité floristique et structurale des cacaoyères du «V baoulé »: Cas de la sous-préfecture de kokumbo (centre, côte d'ivoire). European Scientific Journal 11(36):1857-7881.

Leakey, R.R.B. 1996. Definition of agroforestry revisited, Agroforestry Today 8(1):5-7.

Mangambu, M.J. De D., Aluma, K.J-Y., Ruurd, V.D., Rugenda-Banga, R. Amour, De D., Mushangalusa, K.F., Chibembe, S.A., Ntahobavuka, H.H., Radar, N.B. et Robbrecht, E. 2015. European Scientific Journal 11(15):135-162.

Monssou, E.O., Vroh, B.T.A., Goné, B.Z.B., Adou Yao, C.Y. et N'guéssan, K.E. 2016. Evaluation de la diversité et estimation de La biomasse aérienne des arbres du Jardin Botanique De Bingerville (District d'Abidjan, Côte d'Ivoire). European Scientific Journal 12(6):1857-7881. DOI : 10.19044/esj.2016.v12n6p185

N'Dja, K.J. 2006. Successions secondaires post-culturales en forêt dense semi décidue de sanaimbo (côte d'ivoire) : Nature, structure et organisation fonctionnelle de la végétation Thèse de doctorat, Université Picardi Jules Verus. 232pp.
Ngom, D., Charahabil, M.M., Sarr, O., Bakhoum, A. et Akpo, L.E. 2014. Perceptions communautaires sur les services écosystémiques d'approvisionnement fournis par le peuplement ligneux de la Réserve de Biosphère du Ferlo (Sénégal). Vertigo 14(2):1-18.

Ouattara, D., Vroh, B.T.A., Kpangui, K.B. et N'guéssan, K.E. 2013. Diversité végétale et valeur pour la conservation de la réserve botanique d'Agbaou en création, Centreouest, Côte d'Ivoire. Journal of Animal and Plant Sciences 20(1):3034-3047.

R Core Team. 2019. R: A language and environment for statistical computing. R Foundation for Statistical Computing, Vienna, Austria. URL https://www.Rproject.org/.

Ruf, F.O. 2012. L'adoption de l'hévéa en Côte d'Ivoire. Prix, mimétisme, changement écologique et social. Économie Rurale 4 (330-331):103-124.

Sandor, M.E., Chazdon, R.L. 2014. Remnant trees affect species composition but not structure of tropical second-growth forest. PLoS ONE 9(1):1-7. DOI:10.1371/ journal.pone.0083284

Schroth, G., Faria, D., Araujo, M., Bede, L., Van Bael, S.A., Cassano, C.R., Oliveira, L.C. and Delabie, J.H.C. 2011. Conservation in tropical landscape mosaics: The case of the cacao landscape of southern Bahia, Brazil. Biodiversity and Conservation 20:1635-1654. DOI : 10. 1007/s10531-011-0052-x

Shannon, C.E. 1948. A mathematical theory of communication. Bell System Technical Journal 27:379-423. DOI : 10.1002/j.15387305.1948.tb01338.x

Somboonsuke, B., Wetayaprasit, P., Chernchom, P. and Pacheerat, K. 2011. Diversification of Smallholding Rubber Agroforestry System (SRAS) Thailand. Kasetsart Journal of Social Sciences 32: 327-339. 
Sow, M., Hély, C., M'bow, C. and Sambou, B. 2013. Fuel and fire behavior analysis for early-season prescribed fire planning in Sudanian and Sahelian savannas. Journal of Arid Environments 89(13):84-93. DOI : 10.1016 / j.jaridenv.2012.09.007

Temgoua, L.F., Dongmo, W., Nguimdo, V. et Nguena, C. 2018. Diversité ligneuse et stock de carbone des systèmes agroforestiers à base de cacaoyers à l'Est Cameroun : Cas de la Forêt d'Enseignement et de Recherche de l'Université de Dschang. Journal of Applied Biosciences 122:12274-12286. DOI : $10.4314 /$ jab. v122i1.7

Temgoua, L.F., Momo, S.M.C. et Boucheké, R.K. 2019. Diversité floristique des ligneux des systèmes agroforestiers cacaoyers du littoral Cameroun: Cas de l'Arrondissement de Loum. European Scientific Journal 15 (9):62-83.

Thierry M. 2005. Adapter la conduite des plantations d'hévéa à la diversité des exploitations villageoises (etude de cas au Cameroun). Thèse. Institut National
Agronomique Paris-Grignon, France. 302pp.

Tondoh, E.J., Kouamé, N.F., Guéi, M.A., Sey, B., Koné, W.A. and Gnessougou, N. 2015. Ecological changes induced by full-sun cocoa farming in Côte d'Ivoire. Global Ecology and Conservation 3:575-595. DOI: 10.1016/j.gecco.2015.02.007

Van Wilgen, B., Navashni, G., Izak, P., Smit J. and Sandra, M-F. 2014. The ongoing development of a pragmatic and adaptive fire management policy in a large African savanna protected area. Journal of Environmental Management 132(14):358368. DOI : 10.1016/j.jenvman.2013.11.003 Vroh, B.T.A., Cissé, A., Adou Yao, Y.C., Kouamé, D., Koffi, K.J., Kpangui, K.B. et Koffi, B.J.C. 2015. Relations entre la diversité et la biomasse aérienne des espèces arborescentes dans les agroforêts traditionnelles à base de cacaoyers: Cas de la localité de Lakota (Côte d'Ivoire). African Crop Science Journal 23(4):311326. DOI : 10.4314/acsj.v23i4.2 